\title{
REMARKS ON SOME TAUBERIAN THEOREMS OF MEYER-KÖNIG, TIETZ AND STIEGLITZ \\ D. LEVIATAN
}

Abstract. A general theorem is proved which deduces from a Tauberian condition $T_{1}$ for a summability method, Tauberian condition $T_{2}$ for the summability method. Recent results of MeyerKönig and Tietz and of Stieglitz are special cases.

1. Introduction. Let $\sum_{n=0}^{\infty} a_{n}$ be an infinite series with $s_{n}=\sum_{k=0}^{n} a_{k}$, $n \geqq 0$, its partial sums; and let $V$ be a sequence-to-sequence summability method. A condition on the sequence $\left\{a_{n}\right\}(n \geqq 0)$ is called a Tauberian condition for $V$ if its fulfillment by $\left\{a_{n}\right\}$ together with the existence of $\lim V s$, where $s=\left\{s_{n}\right\}$, implies $\sum_{n=0}^{\infty} a_{n}$ exists and $=\lim V s$.

Recently in a series of papers Meyer-König and Tietz [2], [3], [4] and Stieglitz [5] have shown in different ways, methods by which one Tauberian condition may be deduced from another. They have introduced some new Tauberian conditions as well as obtained some known Tauberian conditions for some of the better known summability methods.

This paper will attempt to bridge the results of Meyer-König and Tietz and those of Stieglitz by showing that all are included in a general theorem. This theorem will also enable us to introduce new Tauberian conditions for well-known methods such as the Borel transform.

Finally we wish to express our thanks to Professor W. MeyerKönig and to Drs. H. Tietz and M. Stieglitz for letting us see their not yet published papers. We follow here Steiglitz' notation.

2. Definitions and main results. Let $S$ denote the sequence-tosequence transformation transforming the sequence $a=\left\{a_{n}\right\}(n \geqq 0)$ into the sequence of partial sums of the series $\sum_{n=0}^{\infty} a_{n}$, i.e. $(S a)_{n}$ $=\sum_{k=0}^{n} a_{k}$; and let $c_{0}, c$ and $m$ denote the space of sequences converging to 0 , converging and bounded, respectively. We shall deal with linear Tauberian conditions; thus they will be given by a linear operator $T$ defined on a subspace of the space of sequences. If $\alpha$ denotes one of the spaces $c_{0}, c$ and $m$, let (see [5])

Received by the editors October 13, 1969.

AMS 1970 subject classifications. Primary 40E05.

Key"words and phrases. Tauberian conditions, summability methods.

Copyright (c) 1971, American Mathematical Society 


$$
\alpha T=\left\{a=\left\{a_{n}\right\}: T a \text { is defined and } \in \alpha\right\},
$$

and finally set $V^{*}=c(V S)$.

The following definition of a Tauberian operator was introduced by Stieglitz [5].

Definition. Let $\alpha$ be one of $c_{0}, c$ and $m$. An operator $T$ is called a Tauberian operator of type $\alpha$ for the method $V$, in short $T^{\alpha}$, if $V^{*} \cap \alpha T \subseteq c S$, with $\lim S a=V-\lim S a, a \in V^{*} \cap \alpha T$.

Our first result generalizes $[5$, Satz 1$]$ and reduces in special cases to results of Meyer-König and Tietz [4].

THEOREM 1. Let $V$ be a regular additive summability method and let $\alpha$ and $\beta$ denote any of $c_{0}, c$ and $m$. Suppose that $T_{1}=T_{1}^{\alpha}$ and that $T_{1}$ possesses a right inverse $T_{1}^{-1}$ satisfying

$$
T_{1}\left(T_{1}^{-1} a\right)=a, \quad a \in \alpha .
$$

Suppose that $A$ and $B$ are two sequence-to-sequence transformations satisfying

$$
\beta \subseteq c B \cap \alpha A
$$

and that $T_{2}$ satisfies

$$
S a=S\left(T_{1}^{-1}\left[A\left(T_{2} a\right)\right]\right)+B\left(T_{2} a\right), \quad a \in V^{*} \cap \beta T_{2} .
$$

Then $T_{2}=T_{2}^{\beta}$.

Proof. First we prove that $V^{*} \cap \beta T_{2} \subseteq c S$. To this end let $a \in V^{*}$ $\cap \beta T_{2}$, then $T_{2} a \in \beta$ and it follows by (2.2) that $B\left(T_{2} a\right) \in c$. Hence by the regularity of $V, V-\lim B\left(T_{2} a\right)$ exists. Since $a \in V^{*}$ and $V$ is additive, we thus conclude by (2.3) that $V-\lim S\left(T_{1}^{-1}\left[A\left(T_{2} a\right)\right]\right)$ exists. Put now $b=T^{-1}\left[A\left(T_{2} a\right)\right]$, then by (2.1) and (2.2), $T_{1} b=A\left(T_{2} a\right) \in \alpha$. Given $T_{1}=T_{1}^{\alpha}$ it follows that $\lim S b$ exists and that $\lim S b=V-\lim S b$, therefore by (2.3) $\lim S a$ exists. Also

$$
\begin{aligned}
\lim S a & =\lim S b+\lim B\left(T_{2} a\right)=V-\lim S b+V-\lim B\left(T_{2} a\right) \\
& =V-\lim \left[S b+B\left(T_{2} a\right)\right]=V-\lim S a .
\end{aligned}
$$

This completes our proof.

We shall derive now the results of Meyer-König and Tietz [4, Sätze 2.1-2.12] from our general theorem. We shall do it briefly and leave the details for the reader.

Let $\left\{\lambda_{n}\right\},\left\{p_{n}\right\}$ and $\left\{q_{n}\right\}$ be sequences with nonzero entries and denote, for $n \geqq 0$,

$$
f_{n}=\frac{q_{n-1}}{p_{n} \lambda_{n}}, \quad g_{n}=\lambda_{n} q_{n}\left(\frac{1}{p_{n} \lambda_{n}}-\frac{1}{p_{n+1} \lambda_{n+1}}\right),
$$




$$
h_{n}=\frac{q_{n}-q_{n-1}}{p_{n}}, \text { and } R=\sum_{n=0}^{\infty}\left|f_{n}-f_{n+1}\right|
$$

(where $q_{-1}=0$ ). If

$$
\left(T_{1} a\right)_{n}=\lambda_{n} a_{n}
$$

and

$$
\left(T_{2} a\right)_{n}=\frac{1}{q_{n}} \sum_{k=0}^{n} p_{k} \lambda_{k} a_{k},
$$

then it follows by $[4,(2.4)]$ that

$$
(S a)_{n}=\sum_{k=0}^{n} \frac{h_{k}}{\lambda_{k}}\left(T_{2} a\right)_{k}+\sum_{k=0}^{n-1}\left(f_{k}-f_{k+1}\right)\left(T_{2} a\right)_{k}+f_{n}\left(T_{2} a\right)_{n} .
$$

Comparing (2.6) with (2.3) we see that $A$ is the diagonal matrix $\operatorname{diag}\left\{h_{n}\right\}$ while $B=\left\|b_{n k}\right\|$ where

$$
\begin{aligned}
b_{n k} & =f_{k}-f_{k+1}, & & 0 \leqq k<n, \\
& =f_{n}, & & k=n, \\
& =0, & & k>n .
\end{aligned}
$$

Now, $B$ is conservative, that is, it transforms $c$ into $c$ if and only if $R<\infty$, and it transforms $m$ into $c$ if and only if $R<\infty$ and $f_{n}=o(1)$. As for $A$, it transforms $c_{0}$ in to $c_{0}$ if and only if $h_{n}=O(1)$, it transforms $c$ into $c$ if and only if $\left\{h_{n}\right\}$ converges, it transforms $m$ into $m$ if and only if $h_{n}=O(1)$, and it transforms $m$ in to $c_{0}$ if and only if $h_{n}=o(1)$.

Using $[4,(2.5)]$, it follows that

$$
(S a)_{n}=\sum_{k=0}^{n} \frac{g_{k}}{\lambda_{k}}\left(T_{2} a\right)_{k}+f_{n+1}\left(T_{2} a\right)_{n} .
$$

In this case we thus have a matrix $A$ which is the diagonal matrix $\operatorname{diag}\left\{g_{n}\right\}$ and $B$ is the diagonal matrix diag $\left\{f_{n+1}\right\}$. So $B$ transforms $c_{0}$ into $c_{0}$ if and only if $f_{n}=O(1)$, it transforms $c$ into $c$ if and only if $\left\{f_{n}\right\}$ converges, and it transforms $m$ into $c_{0}$ if and only if $f_{n}=o(1)$. As for $A$ the cases are similar to those of the above $A$ and obtained by replacing each $h_{n}$ by $g_{n}$.

REMARK. If, in Theorem $1, V$ is assumed to be conservative rather than regular, we can still prove that $V^{*} \cap \beta T_{2} \subseteq c S$ but not that $\lim S a$ $=V$ - $\lim S a$. In fact this conclusion holds even if we assume $V^{*}$ $\cap \alpha T_{1} \subseteq c S$ rather than $T_{1}=T_{1}^{\alpha}$.

A similar theorem whose proof is left to the reader generalizes [5, Satz 2]. 
THEOREM 2. Let $V$ be an additive summability method that transforms $c_{0}$ into $c_{0}$, and let $\alpha$ and $\beta$ be any of $c_{0}, c$ and $m$. Suppose that $T_{1}$ satisfies the requirements of Theorem 1 , that $A$ and $B$ satisfy

$$
\beta \subseteq c_{0} B \cap \alpha A,
$$

and that $T_{2}$ satisfies (2.3). Then $T_{2}=T_{2}^{\beta}$.

As an application one may take the operators $T_{1}$ and $T_{2}$ given by (2.4) and (2.5) respectively. Then the following is an immediate consequence of (2.7) and Theorem 2.

CoROllary 1. Let $V$ be an additive summability method transforming $c_{0}$ into $c_{0}$. Then (i) if $g_{n}=O(1)$ and $f_{n}=O(1)$, and if $T_{1}=T_{1}^{c_{0}}$, then $T_{2}=T_{2}^{c_{0}}$; (ii) if $g_{n}=o(1)$ and $f_{n}=o(1)$, and if $T_{1}=T_{1}^{c_{0}}$, then $T_{2}=T_{2}^{m}$; and (iii) if $g_{n}=O(1)$ and $f_{n}=o(1)$, and if $T_{1}=T_{1}^{m}$, then $T_{2}=T_{2}^{m}$.

REMARK. In Theorem 2 if $V$ is assumed to transform $c_{0}$ into $c$ rather than $c_{0}$ we can still prove that $V^{*} \cap \beta T_{2} \subseteq c S$ but not that $\lim S a=V-\lim S a$. This conclusion holds even if we assume $V^{*} \cap \alpha T_{1}$ $\subseteq c S$ rather than $T_{1}=T_{1}^{\alpha}$.

3. Some new Tauberian conditions. Let $\left\{\lambda_{n}\right\},\left\{p_{n}\right\}$ and $\left\{q_{n}\right\}$ be sequences with nonzero entries and let $T_{1}$ be the operator defined by (2.4). Define an operator $T_{2}$ by taking the transposed matrix of the matrix in (2.5), namely, define

$$
\left(T_{2} a\right)_{n}=p_{n} \sum_{k=n}^{\infty} \frac{\lambda_{k} a_{k}}{q_{k}},
$$

provided the sum on the right-hand side exists. Denote, for $n \geqq 0$,

$$
\begin{gathered}
f_{n}{ }^{\prime}=\frac{q_{n}}{\lambda_{n} p_{n+1}}, \quad g_{n}{ }^{\prime}=\frac{\lambda_{n}}{p_{n}}\left(\frac{q_{n}}{\lambda_{n}}-\frac{q_{n-1}}{\lambda_{n-1}}\right), \\
h_{n}^{\prime}=q_{n}\left[\frac{1}{p_{n}}-\frac{1}{p_{n+1}}\right], \quad \text { and } \quad R^{\prime}=\sum_{n=0}\left|f_{n}^{\prime}-f_{n-1}^{\prime}\right|
\end{gathered}
$$

(where $q_{-1}=0$ ). The following are proved exactly as (2.6) and (2.7). If for $\left\{a_{n}\right\}, T_{2} a$ is defined, then

$$
(S a)_{n}=\sum_{k=0}^{n} \frac{h_{k}^{\prime}}{\lambda_{k}}\left(T_{2} a\right)_{k}+\sum_{k=0}^{n}\left(f_{k}^{\prime}-f_{k-1}^{\prime}\right)\left(T_{2} a\right)_{k}-f_{n}^{\prime}\left(T_{2} a\right)_{n+1}
$$

and

$$
(S a)_{n}=\sum_{k=0}^{n} \frac{g_{k}^{\prime}}{\lambda_{k}}\left(T_{2} a\right)_{k}-f_{n}^{\prime}\left(T_{2} a\right)_{n+1}
$$


Therefore parallel to [4, Sätze 2.1-2.12] we have

TheOREM 3. Let $V$ be a regular additive summability method. Then in what follows if $\left(^{*}\right)$ is satisfied and $T_{1}=T_{1}^{\alpha}$, then $T_{2}=T_{2}^{\beta}$.

$$
\begin{aligned}
& h_{n}^{\prime}=O(1), \\
& h_{n}^{\prime}=o(1), \\
& \left\{h_{n}^{\prime}\right\} \in c, \\
& h_{n}^{\prime}=O(1), \\
& h_{n}^{\prime}=o(1), \\
& h_{n}^{\prime}=O(1), \\
& g_{n}^{\prime}=O(1), \\
& g_{n}^{\prime}=o(1), \\
& \left\{g_{n}^{\prime}\right\} \in c, \\
& g_{n}^{\prime}=O(1), \\
& g_{n}^{\prime}=o(1), \\
& g_{n}^{\prime}=O(1),
\end{aligned}
$$$$
R^{\prime}<\infty \text {, }
$$$$
R^{\prime}<\infty
$$$$
R^{\prime}<\infty \text {, }
$$$$
R^{\prime}<\infty \text {, }
$$$$
R^{\prime}<\infty, f_{n}^{\prime}=o(1)
$$$$
R^{\prime}<\infty, f_{n}^{\prime}=o(1) \text {, }
$$$$
f_{n}^{\prime}=O(1) \text {, }
$$$$
\left\{f_{n}^{\prime}\right\} \in c \text {, }
$$$$
\left\{f_{n}^{\prime}\right\} \in c
$$$$
\left\{f_{n}^{\prime}\right\} \in c \text {, }
$$$$
f_{n}^{\prime}=o(1) \text {, }
$$$$
f_{n}^{\prime}=o(1) \text {, }
$$

$\alpha \quad \beta$

$c_{0} \quad c_{0}$

$c_{0} \quad c$

$c \quad c$

$m \quad c$

$c_{0} \quad m$

$m \quad m$

$c_{0} \quad c_{0}$

$c_{0} \quad c$

$c \quad c$

$m \quad c$

$c_{0} \quad m$

$m \quad m$

A result similar to Corollary 1 can also be proved.

As an application take any $V$ such that $n^{1 / 2} a_{n}=0(1)$ is a Tauberian condition for $V$; for instance the Borel transform is such a method. Then

$$
e^{n^{1 / 2}} \sum_{k=n}^{\infty} e^{-k^{1 / 2}} a_{k}=o(1)
$$

is also a Tauberian condition for $V$. For if $p_{n}=e^{n^{1 / 2}}, q_{n}=n^{1 / 2} e^{n^{1 / 2}}$ and $\lambda_{n}=n^{1 / 2}, n \geqq 0$ (the values for $q_{0}$ and $\lambda_{0}$ are not important), then $h_{n}^{\prime}=O(1)$ and $f_{n}^{\prime} \uparrow 1$, as $n \rightarrow \infty$, whence $R^{\prime}<\infty$. Our result follows now by Theorem 3(i).

Take $V$ such that $\lambda_{n} a_{n}=o(1)$ is a Tauberian condition for $V$. Then if $\lambda_{n}=o(n)$ or $\lambda_{n}=O(n)$, then $\left\{n \sum_{m=n}^{\infty} a_{m} / m\right\}$ converges or $n \sum_{m=n}^{\infty} a_{m} / m=o(1)$, respectively is also a Tauberian condition for $V$. For if $p_{n}=n$ and $q_{n}=n \lambda_{n}$, then $h_{n}^{\prime}=o(1)$ or $h_{n}^{\prime}=O(1)$ respectively and $f_{n}^{\prime} \uparrow 1$, as $n \rightarrow \infty$, whence $R^{\prime}<\infty$. Therefore our results follow by Theorems 3(iii) and 3(i).

As the case $\lambda_{n}=n^{1 / 2}$ shows, it is possible to obtain different Tauberian operators $T_{2}$ even if we start with the same $T_{1}$. We shall give still another Tauberian operator, an operator that was used by us 
[1] while dealing with the quasi-Hausdorff methods. It is related also to the operators defined by $[4,(2.9)]$ and $[5,(9)]$.

Let $T_{1}$ be the operator defined by (2.4) where $\lambda_{n} \neq 0,-1$, $n=0,1,2, \ldots$. Define the operator $T_{2}$ by

$$
\left(T_{2} a\right)_{n}=\sigma_{n} \sum_{k=n}^{\infty} \frac{a_{k}}{\sigma_{k+1}}
$$

where

$$
\begin{aligned}
\sigma_{n} & =1, & & n=0, \\
& =\prod_{m=0}^{n-1}\left(1+\frac{1}{\lambda_{m}}\right), & & n \geqq 1,
\end{aligned}
$$

whenever the sum on the right-hand side of (3.5) exists. It is readily seen that if $T_{2} a$ is defined, then

$$
(S a)_{n}=\sum_{k=0}^{n} \frac{1}{\lambda_{k}}\left(T_{2} a\right)_{k}+\left(T_{2} a\right)_{0}-\left(T_{2} a\right)_{n+1} .
$$

Consequently $A$ is the identity operator while $B=\left\|b_{n k}\right\|$ where

$$
\begin{aligned}
b_{n k} & =1, & & n \geqq 0, \quad k=0, \\
& =-1, & & k=n+1, \\
& =0, & & \text { elsewhere. }
\end{aligned}
$$

Hence $B$ transforms $c_{0}$ into $c$ and $c$ into $c$. The following is an immediate consequence of Theorem 1.

THEOREM 4. Let $V$ be a regular additive summability method and let $T_{1}$ and $T_{2}$ be defined by (2.4) and (3.5) respectively. Then if $T_{1}=T_{1}^{c_{0}}$, then $T_{2}=T_{2}^{c_{0}}$ and if $T_{1}=T_{1}^{c}$, then $T_{2}=T_{2}^{c}$.

For some quasi-Hausdorff methods $T_{1}=T_{1}^{c_{0}}$ and so also $T_{2}=T_{2}^{c_{0}}$ as can be obtained by [1].

4. Conclusion. Let the operator $T_{1}$ be a Tauberian operator of type $\alpha$, for the regular additive method $V$, which possesses a right inverse $T_{1}^{-1}$ satisfying (2.1). Let $\beta$ be one of $c_{0}, c$ and $m$. Then we may ask whether it is possible to find $T_{2}$ a Tauberian operator of type $\beta$ for $V$.

If there exist operators $A$ and $B$ which satisfy (2.2) and such that $\left(S T_{1}^{-1} A+B\right)$ possesses a nontrivial right inverse $\left(S T_{1}^{-1} A+B\right)^{-1}$ with

$$
\left(S T_{1}^{-1} A+B\right)\left[\left(S T_{1}^{-1} A+B\right)^{-1} a\right]=a,
$$

for all $a$ such that $\left(S T_{1}^{-1} A+B\right)^{-1} a \in \beta$, then $T_{2}$ may be defined by

$$
T_{2}=\left(S T_{1}^{-1} A+B\right)^{-1} S \text {. }
$$


For then $T_{2}$ satisfies (2.3) and our result follows by Theorem 1.

Similar remarks can be made if $V$ transforms $c_{0}$ in to $c_{0}$.

\section{REFERENCES}

1. D. Leviatan, Some Tauberian theorems for quasi-Hausdorf transforms, Math. Z. 108 (1969), 213-222. MR 39 \#7327.

2. W. Meyer-König and $\mathrm{H}$. Tietz, On Tauberian conditions of type o, Bull. Amer. Math Soc. 73 (1967), 926-927. MR 35 \#7036.

3. - Über die Limitierungsumkehrsätze vom Typ o, Studia Math. 31 (1968), 205-216. MR 38 \#3654.

4. - Über Umkehrbedingungen in der Limiterungstheorie, Arch. Math. (Brno) 5 (1969), 177-186.

5. M. Stieglitz, Über ausgezeichnete Tauber-Matrizen, Arch. Math. (Brno) 5 (1969), 227-233.

UNIVERSity of IllinoIs, URbANA, IllinoIs 61801 